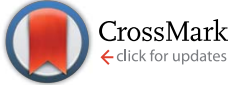

Cite this: RSC Adv., 2016, 6, 110600

Received 8th September 2016 Accepted 12th November 2016

DOI: $10.1039 / c 6 r a 22490 c$

www.rsc.org/advances

\section{Modular supramolecular ureidopyrimidinone polymer carriers for intracellular delivery $\dagger$}

\author{
Maarten H. Bakker, ${ }^{\text {ab }}$ Roxanne E. Kieltyka, ${ }^{\text {ab }}$ Lorenzo Albertazzi $^{\text {ab }}$ \\ and Patricia Y. W. Dankers*abc
}

Ureidopyrimidinone-based polymers in solution provide a new platform for intracellular drug delivery. Here we show the synthesis of functional supramolecular polymers by mixing of monomers with various functionalities. Introduction of cationic monomers made them suitable for cellular uptake and siRNA delivery.

The formulation and intracellular delivery of pharmacologically active substances to target tissues, cells and specific cellular compartments remain important and extremely challenging tasks in the drug delivery research field. ${ }^{1}$ To date, the predominant types of delivery vehicles are based on covalent polymeric nanoparticles and supramolecular liposomal vesicles. ${ }^{2,3}$ Stateof-the-art approaches for drug delivery combine the best of both worlds in the latest generation of hybrid lipid-polymer nanoparticles.,5 Likewise, supramolecular polymers and complexes based on directional non-covalent interactions merge characteristics of both classes and are currently being explored for several biomedical applications. ${ }^{6-9}$ Some examples of supramolecular polymers include materials based on discotic benzene tricarboxamides, ${ }^{\mathbf{1 0}}$ porphyrins, ${ }^{\mathbf{1 1}}$ carbohydrate conjugated aromatics, ${ }^{12}$ and peptide amphiphiles. ${ }^{13}$ However, few examples exist on the use of 1D supramolecular polymers for use in intracellular delivery, ${ }^{\mathbf{1 4 - 1 6}}$ while they can serve as a potentially attractive platform. A frequently reported supramolecular building block that proved to be suitable as biomaterial is the ureidopyrimidinone (UPy) unit, ${ }^{17}$ that can dimerize via a self-complementary quadruple hydrogen bonding motif. Polymers functionalized with UPy-groups are able to selfassemble into columnar stacks via hydrogen bonds and

${ }^{a}$ Institute for Complex Molecular Systems, Eindhoven University of Technology, P. O. Box 513, 5600 MB Eindhoven, The Netherlands. E-mail: p.y.w.dankers@tue.nl

${ }^{b}$ Laboratory of Chemical Biology, Eindhoven University of Technology, P. O. Box 513, 5600 MB Eindhoven, The Netherlands

${ }^{c}$ Laboratory for Cell and Tissue Engineering, Eindhoven University of Technology, P. O. Box 513, 5600 MB Eindhoven, The Netherlands

$\dagger$ Electronic supplementary information (ESI) available: Materials \& methods, synthesis of monomers. See DOI: 10.1039/c6ra22490c additional $\pi-\pi$ interactions. ${ }^{18}$ In our group, UPy moieties have been employed to produce a range of supramolecular biomaterials; from relatively static solid-like bioactive films and membranes, ${ }^{19}$ to more dynamic, injectable and stimuliresponsive hydrogels. ${ }^{\mathbf{2 0 2 1}}$ Here, we report the preparation of multicomponent functional supramolecular polymers in aqueous solution, and demonstrate the capability for cellular uptake and siRNA complexation and delivery (Fig. 1).

Structurally related monomers were designed and synthesised. In general the monomers contain the supramolecular UPy unit, a hydrophobic spacer to shield hydrogen bonding in the core of the stack, and a readily functionalizable water soluble oligo ethylene glycol (OEG) linker. Amine functionalized compounds were synthesized to obtain cationic monomers (1), that can be mixed with neutral acetamide functionalized monomers (2) and fluorescently labelled monomers (3) (Fig. 1a). The monomers were separately dissolved and stored in $\mathrm{MeOH}$.

Supramolecular polymerization can be initiated by injection of the concentrated $\mathrm{MeOH}$ stock solutions in aqueous solution. Multicomponent supramolecular polymers were formed by injection of the different UPy monomers in various ratios. siRNA can be condensed via the cationic charges, either during supramolecular polymerization or after the supramolecular polymer has formed. Subsequently, the siRNA-polymer complexes can be delivered to cells (Fig. 1b).

Polymer formation was probed with the hydrophobic dye nile red, which is strongly fluorescent upon encapsulation in a hydrophobic compartment. ${ }^{22}$ Self-assembled UPy polymers were evaluated with varying amounts of amine monomers; from $0 \%$ (neutral), $20 \%$ cationic, $50 \%$ cationic, $80 \%$ cationic, to $100 \%$ cationic monomer (cationic). For all prepared polymers an increase of NR fluorescent intensity was observed, which indicates aggregate and hydrophobic pocket formation in all samples (Fig. 2a). The inverse correlation between the cationic monomer percentage and NR fluorescent intensity reveals a decreased presence or accessibility of hydrophobic compartments upon increased amine content. 
a)
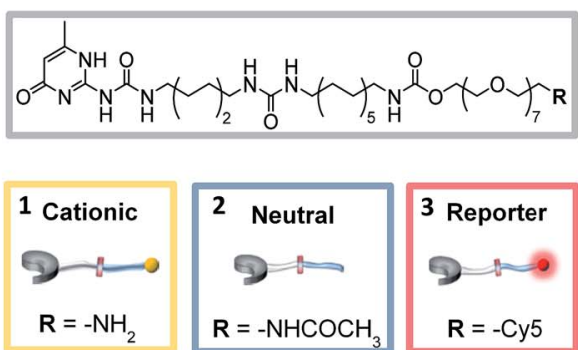

b)

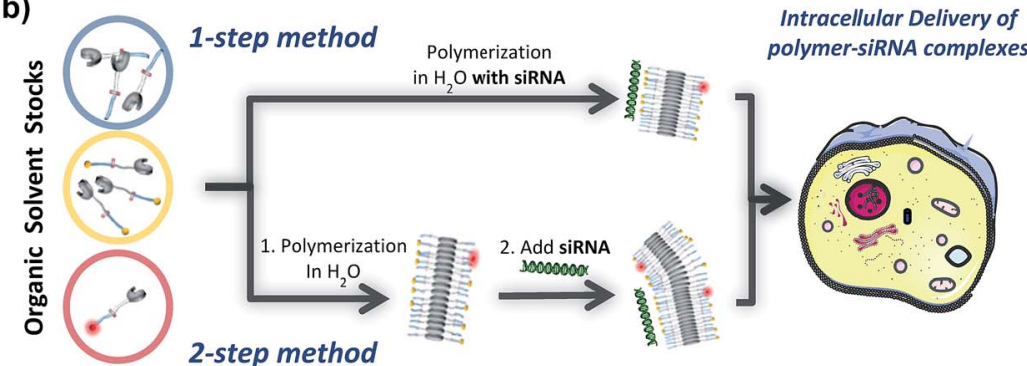

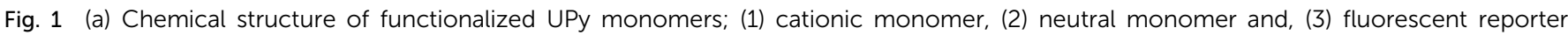

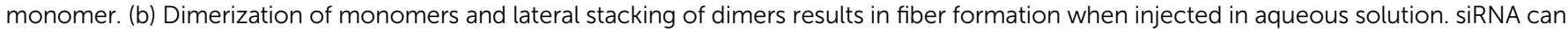

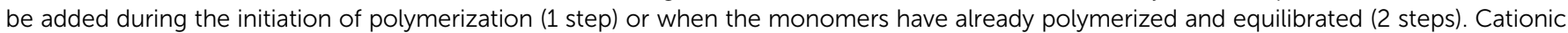

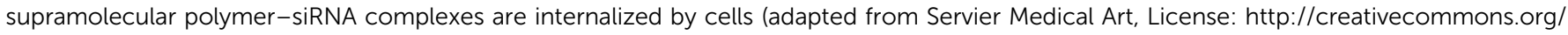
licenses/by/3.0/legalcode).

a)

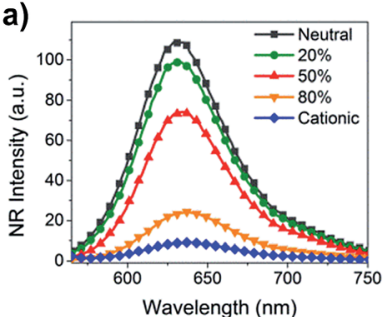

c)

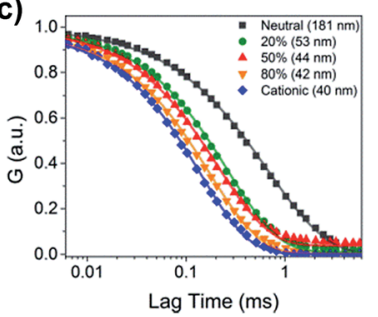

b)

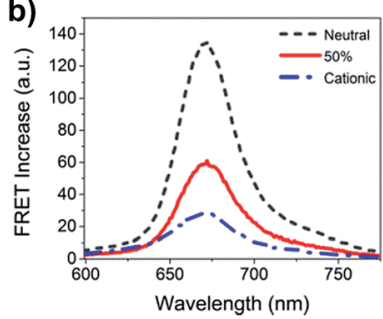

d)

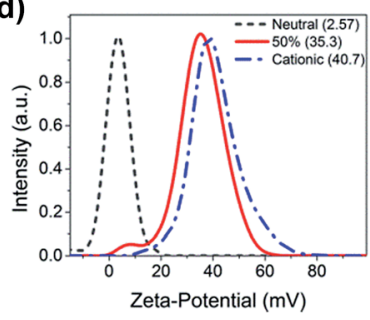

Fig. 2 (a) Nile Red (NR) encapsulation. (b) FRET increase between NR and Cy5. (c) Correlograms (symbols) and stretched exponential decay fits (lines) of DLS measurements (angle $102^{\circ}$ ). Hydrodynamic radius in legend. (d) Zeta potentials measured on three different UPy fiber compositions. Average in legend (mV).

Next, all prepared supramolecular polymers were coassembled with $1 \%$ of the reporter monomer to enable fluorescent imaging and characterization. In order to verify the incorporation of the reporter monomer in the aggregate, FRET was measured between encapsulated NR and the Cy5 of the reporter monomer (Fig. 2b). After NR excitation, strong Cy5 emission showed that the fluorophores are in close proximity. This serves as a strong indication of the incorporation of the fluorescent monomer in the aggregate and demonstrates the potential of our approach to incorporate functionalities into supramolecular polymers. Multi-angle dynamic light scattering (DLS) showed a decrease in aggregate size when cationic monomers were incorporated (Fig. 2c). Cumulant analysis on a full range of measured angles gave a hydrodynamic radius of $181 \mathrm{~nm}$ for a neutral polymer, which decreased to $40 \mathrm{~nm}$ for a fully cationic polymer. Interestingly, this decrease in size correlates with the data from the nile red experiments. A reduced availability of hydrophobic compartments upon co-

assembly of cationic monomers can be explained by the existence of smaller aggregates. One reason for this decrease in size is hypothesized to be the increase in electrostatic repulsive interactions within a single polymer. This concept has previously been employed to control growth and size of supramolecular polymers. ${ }^{23}$

Next, zeta potential measurements showed positive values of $35.3 \mathrm{mV}$ and $40.7 \mathrm{mV}$ for a $50 \%$ and a fully cationic polymer respectively, while $2.57 \mathrm{mV}$ was measured for the neutral polymer (Fig. 2d). An equivalent ratio of neutral and cationic monomers seems nearly sufficient to saturate the periphery of the supramolecular polymer with charges, based on the slight increase in the zeta potential on going from $50 \%$ to full saturation with cationic polymer. Notably for the $50 \%$ polymer, the presence of one major zeta potential distribution indicates that most of the monomers have co-assembled into a single heterogeneous polymer. This key question has to be resolved for every new or distinct monomer that one wishes to co-assemble. Generally, a high flexibility in functionalization is expected owing to the robust self-assembling UPy unit and nearly identical monomeric structure. Nonetheless, small alterations such as charge incorporation can greatly influence the properties of the final composition, and therefore, spontaneous co-assembly of any monomer is not self-evident.

We expected that cationic supramolecular UPy polymers are able to bind cell membranes and be internalized, even if the polymers consist partially of neutral monomers. Cellular internalization and cell viability studies were conducted with human kidney (HK-2) cells and confocal microscopy. The 50\% and fully cationic polymers were clearly located on the cellular membrane after 5 minutes of incubation (Fig. 3a). No significant difference in binding was observed between these distinctly charged polymers. When neutral fibers were administered no cell binding or permeation was observed. It means that positive charges on the supramolecular polymer are necessary to bind to the membrane and internalize. After 4 hours of incubation, UPy polymer internalization and cell viability was visualized. Both polymers that initially bound to the membrane were internalized by the cells and primarily located in the perinuclear region. A LIVE/DEAD staining showed that internalization of supramolecular UPy polymers did not 
a)
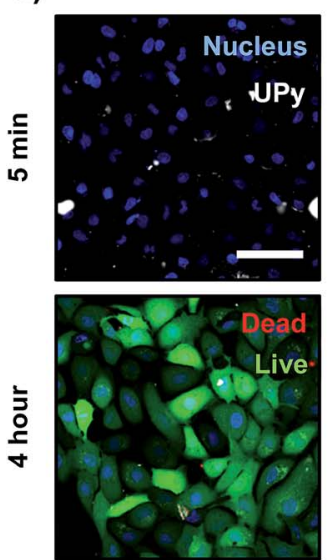

$50 \%$
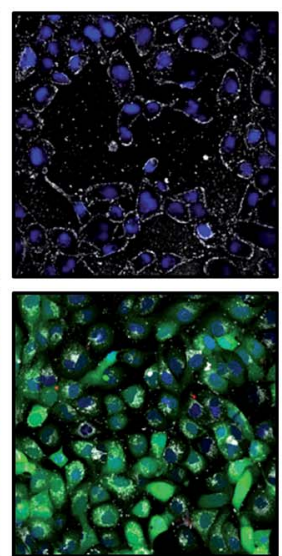

Cationic
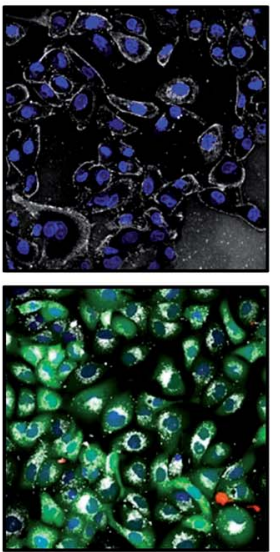

b)

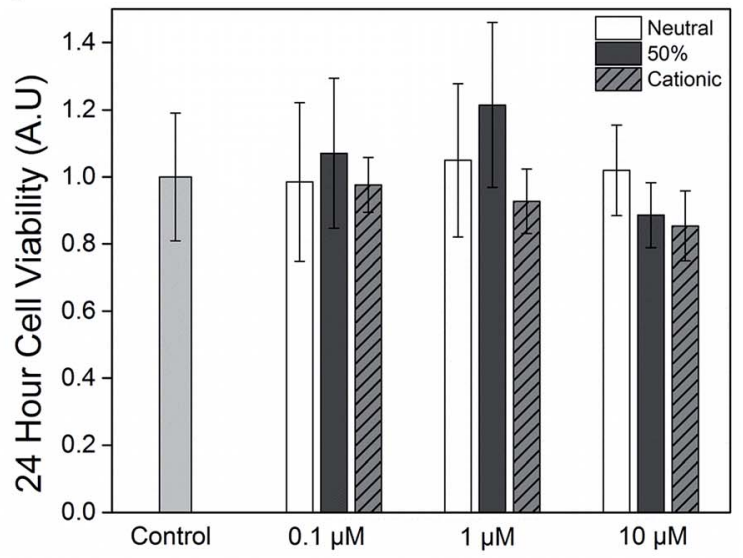

Fig. 3 (a) Confocal microscopy images of live HK-2 cells incubated with $10 \mu$ M UPy polymers after 5 minutes and after 4 hours with a LIVE/DEAD assay. Supramolecular UPy polymers are visible due to incorporation of $1 \%$ reporter monomer. Scale bar represents $100 \mu \mathrm{m}$. (b) MTT assay on HK-2 cells incubated with UPy aggregates for 24 hours $(p>0.05)$.

negatively affect cell viability. Furthermore, an MTT assay with polymer concentrations up to $10 \mu \mathrm{M}$ demonstrated that the polymers did not affect metabolic activity of HK-2 cells 24 hours after incubation (Fig. 3b).

The excellent internalization of cationic supramolecular UPy polymers renders them suitable for applications in intracellular drug delivery. One of the advantages of supramolecular polymers is their facile preparation. The robustness of this approach was challenged during siRNA complex formation. Commonly, preparation of these complexes is a 2-step process that involves first synthesising the carrier, and subsequently siRNA complexation. Here, a 1-step formulation was also employed by injection of all monomers and siRNA in aqueous solution simultaneously. Thus, the capacity of the supramolecular polymerization to occur in the presence of siRNA was also evaluated. The $50 \%$ and fully cationic polymers at various $\mathrm{N} / \mathrm{P}$ ratios (ratio of moles of amine in carrier to moles of phosphate in siRNA) were used for siRNA complexation. Agarose gel retardation assays showed that siRNA was completely condensed from N/P ratios of 10 and higher, independent of exact supramolecular polymer composition or formulation technique (Fig. 4a). This result demonstrates that pre-formation of the polymers is not required to form stable complexes with siRNA. Nonetheless, DLS showed that a)
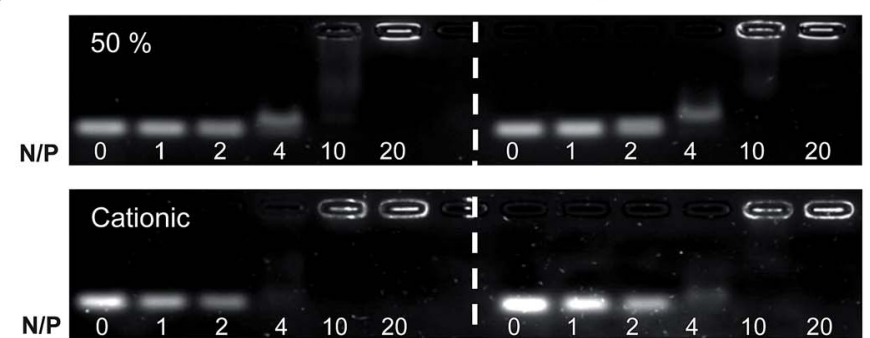

b)

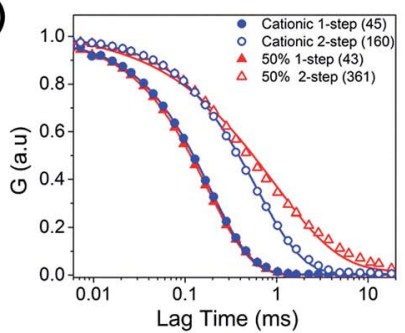

d)

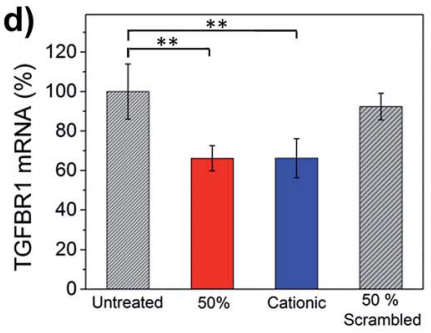

c)
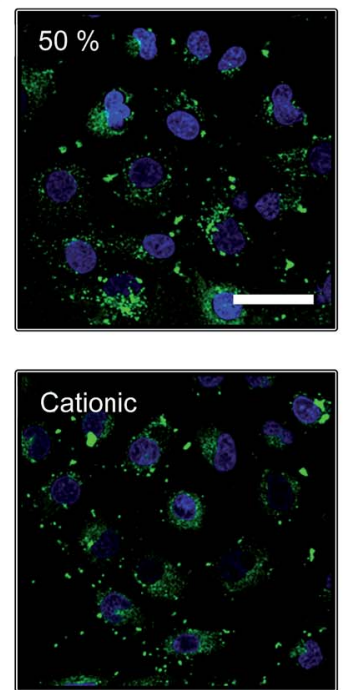

1-step formulation
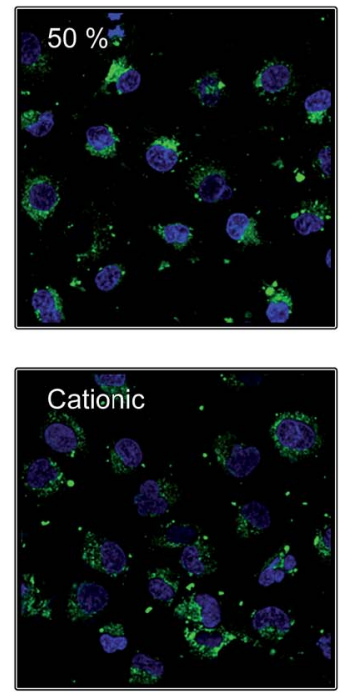

Fig. 4 (a) Agarose gel retardation assay on UPy-siRNA complexes, prepared via two formulation methods. (b) Correlograms (symbols) and stretched exponential decay fits (lines) of DLS measurements (angle $102^{\circ}$ ) on UPy-siRNA complexes. Hydrodynamic radius in legend (nm). (c) Internalization of siRNA in HK-2 cells after 2 hour incubation with UPy-siRNA complexes. Nucleus is stained in blue, siRNA fluoresces green from covalently bound Alexa Fluor 488. Scale bar represents $50 \mu \mathrm{m}$. (d) TGFBR1 mRNA expression of HK-2 cells treated with UPy polymers complexed with TGFBR1 siRNA via the 1-step formulation method, and a scrambled siRNA as negative control. 
complexes prepared via 2-step formulation were different in structure. They exhibited significantly higher hydrodynamic radii (361 and $160 \mathrm{~nm}$ for the 50\% and fully cationic polymers, respectively), compared to the 1-step prepared complexes (43 and $45 \mathrm{~nm}$ ) (Fig. 4b). This observation shows that the process of supramolecular polymerization can be influenced by the presence of opposite charged molecules. This behaviour is possibly explained by a templating effect of the siRNA, similar to what has been reported for other systems. ${ }^{24}$

Next, HK-2 cells were treated with UPy-siRNA complexes prepared via both methods, and internalization of the fluorescently labelled siRNA after two hours incubation was studied. Both the $50 \%$ and fully cationic polymers demonstrated the ability to transfect siRNA into cells (Fig. 4c). No clear difference was observed in amount of internalized siRNA or in intracellular location. Naturally, this may indicate that complex structure is not a critical parameter for the delivery, or is nullified when cells come into play. Lastly, a functional siRNA that targets transforming growth factor beta receptor 1 (TGFBR1) mRNA was transfected to determine the silencing efficacy for a $50 \%$ and full cationic polymer at N/P ratio 10 prepared via convenient 1-step formulation (Fig. 4d). Transfection of siRNA with both polymers resulted in a $34 \%$ reduction of mRNA expression; proving the functionality of the delivered siRNA, and at the same time the feasibility of this supramolecular approach.

In conclusion, we showed the use of the UPy motif to formulate supramolecular polymers with various functionalities diluted in aqueous solution. Here we demonstrated the applicability of this concept by preparing polymers suitable for cellular internalization and intracellular delivery of siRNA. In principle this approach can also be applied for research in other biomedical areas such as bioimaging and biosensing.

\section{Acknowledgements}

We thank the Ministry of Education, Culture and Science (Gravity program 024.001.035), the European Research Council (FP7/2007-2013) ERC Grant Agreement 308045, and the Netherlands Organization for Scientific Research (NWO - VENI Grant: 722.014.010) for funding.

\section{References}

1 N. Martinho, J. Biomater. Nanobiotechnol., 2011, 2, 510-526. 2 W. B. Liechty, D. R. Kryscio, B. V. Slaughter and N. A. Peppas, Annu. Rev. Chem. Biomol. Eng., 2010, 1, 149-173.

3 T. M. Allen and P. R. Cullis, Adv. Drug Delivery Rev., 2013, 65, 36-48.

4 S. Tan, X. Li, Y. Guo and Z. Zhang, Nanoscale, 2013, 5, 860-872.
5 K. Raemdonck, K. Braeckmans, J. Demeester and S. C. D. Smedt, Chem. Soc. Rev., 2013, 43, 444-472.

6 R. Dong, Y. Zhou, X. Huang, X. Zhu, Y. Lu and J. Shen, Adv. Mater., 2015, 27, 498-526.

7 K. Petkau-Milroy, M. H. Sonntag and L. Brunsveld, Chem.Eur. J., 2013, 19, 10786-10793.

8 M. J. Webber, E. A. Appel, E. W. Meijer and R. Langer, Nat. Mater., 2016, 15, 13-26.

9 G. Yu, D. Wu, Y. Li, Z. Zhang, L. Shao, J. Zhou, Q. Hu, G. Tang and F. Huang, Chem. Sci., 2016, 7, 3017-3024.

10 P. Besenius, J. L. M. Heynens, R. Straathof, M. M. L. Nieuwenhuizen, P. H. H. Bomans, E. Terreno, S. Aime, G. J. Strijkers, K. Nicolay and E. W. Meijer, Contrast Media Mol. Imaging, 2012, 7, 356-361.

11 M. Sun, H.-Y. Zhang, B.-W. Liu and Y. Liu, Macromolecules, 2013, 46, 4268-4275.

12 D.-W. Lee, T. Kim, I.-S. Park, Z. Huang and M. Lee, J. Am. Chem. Soc., 2012, 134, 14722-14725.

13 S. Khan, S. Sur, P. Y. W. Dankers, R. M. P. da Silva, J. Boekhoven, T. A. Poor and S. I. Stupp, Bioconjugate Chem., 2014, 25, 707-717.

14 D. Missirlis, H. Khant and M. Tirrell, Biochemistry, 2009, 48, 3304-3314.

15 M. H. Bakker, C. C. Lee, E. W. Meijer, P. Y. W. Dankers and L. Albertazzi, ACS Nano, 2016, 10, 1845-1852.

16 K. Petkau-Milroy, M. H. Sonntag, A. H. A. M. van Onzen and L. Brunsveld, J. Am. Chem. Soc., 2012, 134, 8086-8089.

17 P. Y. W. Dankers, M. C. Harmsen, L. A. Brouwer, M. J. A. Van Luyn and E. W. Meijer, Nat. Mater., 2005, 4, 568-574.

18 R. P. Sijbesma, F. H. Beijer, L. Brunsveld, B. J. B. Folmer, J. H. K. K. Hirschberg, R. F. M. Lange, J. K. L. Lowe and E. W. Meijer, Science, 1997, 278, 1601-1604.

19 B. B. Mollet, M. Comellas-Aragonès, A. J. H. Spiering, S. H. M. Söntjens, E. W. Meijer and P. Y. W. Dankers, J. Mater. Chem. B, 2014, 2, 2483-2493.

20 M. M. C. Bastings, S. Koudstaal, R. E. Kieltyka, Y. Nakano, A. C. H. Pape, D. A. M. Feyen, F. J. van Slochteren, P. A. Doevendans, J. P. G. Sluijter, E. W. Meijer, S. A. J. Chamuleau and P. Y. W. Dankers, Adv. Healthcare Mater., 2014, 3, 70-78.

21 M. Guo, L. M. Pitet, H. M. Wyss, M. Vos, P. Y. W. Dankers and E. W. Meijer, J. Am. Chem. Soc., 2014, 136, 6969-6977. 22 P. Greenspan and S. D. Fowler, J. Lipid Res., 1985, 26, 781-789. 23 J. Baram, E. Shirman, N. Ben-Shitrit, A. Ustinov, H. Weissman, I. Pinkas, S. G. Wolf and B. Rybtchinski, J. Am. Chem. Soc., 2008, 130, 14966-14967.

24 Y. Ruff, T. Moyer, C. J. Newcomb, B. Demeler and S. I. Stupp, J. Am. Chem. Soc., 2013, 135, 6211-6219. 